American Journal of Applied Sciences 4 (1): 49-52, 2007

ISSN 1546-9239

(C) 2007 Science Publications

\title{
Analysis of Motion of Partial System on Phase Plane and Determination of Stable Cutting Regions
}

\author{
Firas Al Qura'n \\ Department of Mechanical Engineering, Tafila Technical University, P.O. Box 179, Tafila 66110, Jordan
}

\begin{abstract}
Turning is one of the most efficient preferred and cutting processes currently known. It was a challenging task for researchers to study the different dynamic properties explores its special dynamical properties, including conditions that insure the stability of the cutting process and the associated nonlinear vibrations. The main goal of this study was to develop and study a simple model, which describe the dynamic interactions in the uncoupled machine tool and cutting process system. A general one-degree-of-freedom mathematical model of orthogonal metal turning was established and examined.
\end{abstract}

Key words: Technological system, movement, stability, graphic methods, phase plane

\section{INTRODUCTION}

The principle directions of development in machine-building technology are related with further improvement of parts quality and acceleration of growth rates of production efficiency.

The perspective method for solving the posed problems is the use of adaptive self-adjusted systems and devices during turning and the other operations. These devices are based on the automatic control of the cutting process; this will ensure an improvement in the mechanical working accuracy by means of the dynamic setting of the machine tool during the process. The later can be achieved by stabilizing the cutting forces and employing a mechanism to correct the elastic displacement (movement) for the used technological systems.

As a rule, all cutting anal devices have a lowered rigidity and oscillating stability. This makes it difficult when such devices are employed in metalworking.

Large relative vibrations between the tool and the workpiece in a metal cutting process can compromise the productivity and accuracy of the used manufacturing technique. This is particularly dangerous when a sudden and uncontrolled rise of vibration amplitude occurs. In many practical situations, the conditions in which such an instability appears can be explained an adequately by linear dynamics derived model. However, more comprehensive insight can be gained only if the dynamic interactions between the machine tool and the cutting process are treated as a nonlinear model. An example of such behavior is selfexcited oscillations, so-called chatter ${ }^{[1]}$.

Several investigations into nonlinear dynamic have shown an existence and importance of chaotic motion occurring in machining. The studies conducted by
Garbec $^{[2]}$, Wiercigroch ${ }^{[3]}$, Wiercigroch and Cheng ${ }^{[4]}$ and Foong et $a l^{[5]}$ are good examples of those investigations. The proposed models have shown the evidence of chaotic vibrations, which are mainly due to the nonlinearity of the dry friction and the intermittent contact between the cutting tool and the workpiece.

The movement stability of the technological system under the effect of external disturbances is determined by the system behavior near the equilibrium position. If the system, under the effect of arbitrarily small initial disturbance, is removed from the equilibrium position then the system is declared unstable.

For the systems used in of metal cutting machines, there are stationary and oscillatory (vibrating) positions of equilibrium. The cutting parameters (speed, feed and depth of cut) are constant in the stationary type of equilibrium. If the cutting parameters are periodically changed, then the positions of equilibrium well vibrate in the system.

During parts processing using metal cutting machines, it is necessary to active a stationary position of equilibrium; which is obtained mostly by eliminating the self-vibrating motion. The self-vibrating motion can be eliminated either by the suitable selection of cutting parameters, or by the means of additional damping systems related with employing special amortizators of energy (dampers). The use of dampers complicates the technological system and, however this is not always economical justified.

Both analytical and graphic methods for determination of the stable motion of the technological system are used for selecting the rational cutting parameters.

Finding the analytical solution of the differential equation, if it is possible, gives in most cases a great 
advantage. The analytical solution is obtained in an algebraic form and does not require the introduction of numerical values of the process parameters or the initial conditions. Once, the solution is obtained, it is possible to set any numerical values and investigate the totality of the solutions that described by the differential equation.

However, it should be kept in mind that only a few of equations that describe the real system are simple enough and permit accurate solution. In general there is no regular methods of integration that can give accurate solution for arbitrarily nonlinear differential equations.

Therefore, using the different types of approximating methods can provide a unique (single) suitable technique for solving the frequently encountered classes of nonlinear equations. Such methods are: small-parameter method $^{[6]}$, average $\operatorname{method}^{[7,8]}$, energetic method ${ }^{[9]}$ and the harmonicbalance method.

The graphic methods of analysis are one of the important methods used for investigating different phenomenon's related with nonlinear vibration. With the help of graphic methods, the solutions of differential equations are sought in the form of integral curves on the phase-plane.

The isoclines, Lenarion and Delta methods can be refereed to the graphic methods used for solving the nonlinear differential equations. The isoclines method is the most broad. This method gives the opportunity to investigate the field of the integral curves with the help of graphics without solving the differential equation. This method is especially valuable when the accurate mode solution for the differential equation is not known. The principle demerit of isoclines method is the relatively long time required.

Lenarion method is particularly convenient for investigating the self-vibrating and also in other cases when the regenerating elastic force is linear with respect to the movement. This method does not require the approximation of the nonlinear characteristic and this is the principle merit of this method. The experimental characteristic may be used for constructing (tracing) the integral curves.

The employment of isoclines and Lenarion methods for obtaining the graphic solution of the differential equation needs to fill all the phase-plane with segments of a straight line that define the direction of the integral curves. Practically if it is necessary to find only one integral curve, then some of these segments are used directly. In these cases the Deltamethod is the most direct straight method for finding the required solution because with the help of this method we can get only the data which is related directly with the interested integral curve.

Stability analysis: When the turning operation is carried out with the help of an adaptive system, the followings are arise: the necessity in analyzing the

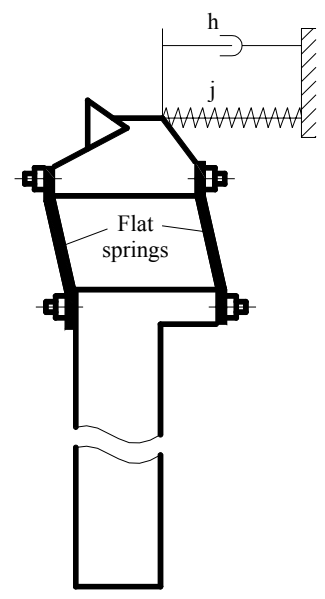

Fig. 1: The limiting vibrating system: the mechanical adaptive system in X-direction. The parameters of the oscillating contour of this system have the following values: $\mathrm{j}=6400$ $\mathrm{N} / \mathrm{m}, \quad \mathrm{m}=18.91 \mathrm{~N}, \quad \Delta=0.035, \mathrm{~h}=123.3$ N.sec/m

oscillating motion of the technological system, the necessity of finding the stable cutting regions and determining the cutting parameters which ensure the non- oscillating turning.

To analyze the oscillating motions of the technological system, it is necessary to define the designed (rated) mathematical model of the system and determine its parameters such as rigidity $(\mathrm{j})$, natural frequency (f), reduced mass (M), logarithmic decrement $(\Delta)$ and the proportional coefficient between the resistance force and the speed $(\mathrm{h})^{[10]}$.

Determination the limiting oscillating system: In connection with the adaptive system based on the compensation of the elastic deformations, the designed model has 9 degrees of freedom: 3 for the lathe spindle in $\mathrm{X}, \mathrm{Y}$ and $\mathrm{Z}$ directions; 3 for the tool post; and 3 for the proposed adaptive system itself. As known in the theory of vibration, the established degrees of freedom are related to the limiting frequencies of the system. This means that the other degrees of freedom can be neglected.

In connection with the considered system, it was established that the limiting frequency equaled to $93 \mathrm{~Hz}$ this corresponds to the natural frequency of the cutting tool (proposed adaptive system) in the direction of the axial component of the cutting force. Therefore, the mathematical model may be represented by a system with one degree of freedom in X-direction (Fig. 1).

To determine the stable cutting conditions, the characteristics of the axial component of the cutting force were established. These linear characteristics were defined experimentally with the help of an arrangement mounted on the base of rigid Sigma 2600 
engine lathe. The axial cutting forces arising during processing were fixed by YDM-600 dynamometer.

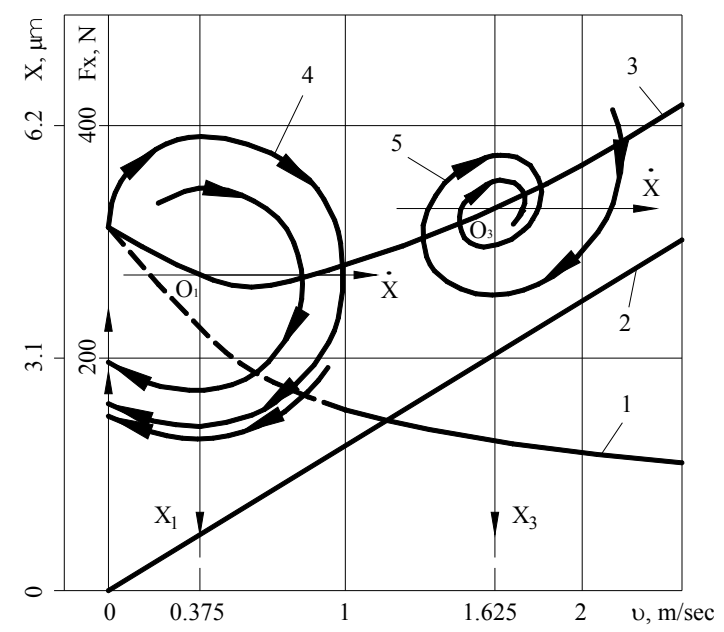

Fig. 2: Phase portrait created by turning with the developed system at $\mathrm{v}=0.375$ and $1.625 \mathrm{~m} / \mathrm{sec}$, $\mathrm{S}=0.07 \mathrm{~mm} / \mathrm{rev}$, and $\mathrm{t}=1.5 \mathrm{~mm}$

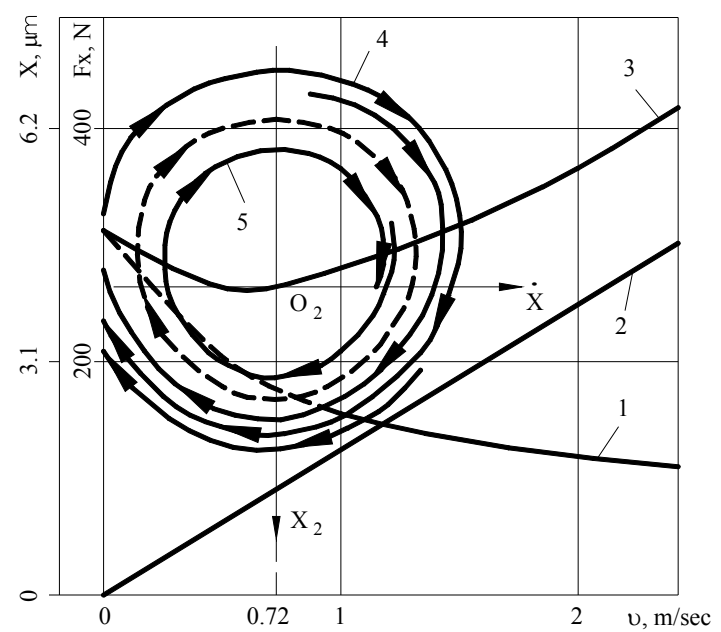

Fig. 3: Phase portrait created by turning with the developed system at $\mathrm{v}=0.72 \mathrm{~m} / \mathrm{sec}, \mathrm{S}=0.07$ $\mathrm{mm} / \mathrm{rev}$, and $\mathrm{t}=1.5 \mathrm{~mm}$

Cutting conditions, workpiece and tool materials: Turning trials were carried on short rigid shaft made from steel 1045 with the following conditions: depth of cut ( $\mathrm{t}$ ) changed in the limits of $0.5 \ldots 1.5 \mathrm{~mm}$, feed rate (S) -0.07 to $0.21 \mathrm{~mm} / \mathrm{rev}$ and cutting speed (v) changed from 1 to $3 \mathrm{~m} / \mathrm{sec}$.

The proposed adaptive system used in this study was provided with triangular cemented carbide insert with main approach angle equaled to $93^{\circ}$, side relief angle equaled to $5^{\circ}$ and main rake angle equaled to $5^{\circ}$.

As a result of the conducted experiments, the nonlinear relationship of the axial force Fx to the speed was obtained at the fixed values of feed rate and depth of cut. These characteristics were correspond to the characteristic of first order since they have a dropping region (Fig. 2).
In order to keep the analysis clear, we will consider the stability of the one-degree-of-freedom model for the cutting process (Fig. 1). The use of the Lenarion method, which is the most broadly used for analyzing the self-vibrating motion of the technological systems, we will present for a turning operation of steel 1045 with the help mechanical adaptive system. Number 1 in Fig. 2 and 3 present the characteristic of cutting force.

Number 2 in the figures refers to the resistant force in the system and number 3 indicates to the distribution characteristic obtained by addling characteristic 1 and 2 .

Constructing the integral curve for different cutting parameters was achieved for the distribution characteristic obtained in the direction of action of the axial component of cutting force Fx.

When selecting the work point on the dropping (falling, incident) section of the distribution characteristic (Fig. 1-3), the integral curve untwists from the unstable singular point $\mathrm{o}_{1}$ and aims for the stable limiting (cluster) 4. Here, a stationary selfvibrating motion, which corresponds to light excitation, is establish in the system. Thus, at the existence of selfvibrating, the cutting parameters will be changed with a frequency equaled to $93 \mathrm{~Hz}$ in this case.

If the work point is selected on the raised section near to the incident section, then too regimes are established in the technological system, stable and selfvibrating.

When selected the work point on the raised section with clearly expressed positive resistance, the integral curve 5 will be twist to the steady singular point $\mathrm{O}_{3}$. This indicates to the lade of the self-vibrating motion. The technological system in this case has steady stationary position of equilibrium and the cutting parameters used will be not changed.

By analyzing the movement of the technological system on the phase plane for all the ranges for the cutting parameters, it is possible to define the steady regions and their corresponding cutting parameters.

Thus, the light and stringent excitation of selfvibrating motion may be occurring in the technological system. The stationary vibrating motion corresponding to light excitation occurs at the dropping sections when a negative resistance is prevail in the system.

The stringent excitation of self-vibrating or the steady stationary position of equilibrium may occur at the raised sections of distribution characteristic.

As previously mentioned, the integral curves on the phase-plane gives descriptive presentation about the character of the system movement at particular values of the system parameters, for evaluating the total of phenomenon's that is related to variation in this or other system-parameter, it is advisable to use the analytic method. The Later needs to construct the so-called bifurcation diagram. During the last two decades, many work about bifurcation theory appeared ${ }^{[11-15]}$. However, little is known about bifurcations of cutting 
manufacturing systems. The use of this method will be considered in forthcoming papers.

\section{CONCLUSION}

Conclusions drawn from turning steel with the help of mechanical adaptive system are as follows:

1. As a result for the investigation in the dynamic characteristics of the components of the turning technological adaptive system, a mathematical model of complex "machine tool + mechanical adaptive system" was obtained and presented as a system with one degree of freedom in the direction of X-axis.

2. The behavior of the adaptive system was investigated successfully using a graphic method during turning of steel.

3. Zones of stable non-vibrational processing were determined.

\section{REFERENCES}

1. Wiercigroch, M. and A.M. Khrivtsov, 2001. Frictional chatter in orthogonal metal cutting. Phil. Trans. Royal Society Land,A: 713-738.

2. Grabec, I.,1988. Chaotic dynamics of the cutting process. Int. J. Mech. Tools Manufact., 28: 19-32.

3. Wiercigroch, M.,1997. Chaotic vibrations of a simple model of the machine tool-cutting process system. Trans. ASME J. Vib. Acoust., 119: 468475.

4. Wiercigroch, M and A. Cheng, 1997. Chaotic and stochastic of metal cutting process. Chaos, Solutions and Fractals, 8: 715-726.
5. Foong, C.H., M. Wiercigroch and W.F. Deans, 2001. Experimental study of the nonlinear dynamics of orthogonal metal cutting. Int. J. Mech. Mech. Engg 4 (In press).

6. Andronov, I., A. Veet and E. Haiken, 1959. Theory of Vibration. Moscow, USSR, pp: 915.

7. Bulgakov, B., 1954. Vibration. Moscow, USSR, pp: 892.

8. Obmorshov, A., 1965. Introduction in Theory Of Vibration. Moscow, Nayka, USSR, pp: 276.

9. Teodorchek, A., 1952. Self- Vibrating Systems. Moscow, Nayka, USSR, pp: 270.

10. Murashkin, S.L. and L.S. Murashkin, 1977. Applied Nonlinear Mechanics of Machine Tools. Leningrad: Mashinostroene.

11. Guckenheimer, J. and P Holmes, 1983. Nonlinear Oscillations, Dynamical Systems and Bifurcation of Vector Fields. Appl. Math Sci., 42: SpringerVerlag

12. Hagedorn, P., 1988. Nonlinear oscillations. Oxford Eng Sci Series 10 Oxford.

13. Kuznetsov, Y.A., 1995. Elements of Applied Bifurcation Theory. Appl. Math. Sci., 112 New York

14. Parker, T.S. and L.O. Chusa, 1989. Practical Numerical Algorithms for Choatic Systems. New York: Springer-Verlag

15. Seydel, R., 1994. Practical bifurcation and stability analysis from equilibrium to chaos. Interdisciplinary App. Math. 